\title{
Hazard grouping of nanomaterials for daphnia and algae toxicity: lessons learned from scientific projects for regulatory applicability
}

\author{
Kathrin Schwirn * ${ }^{*}$ and Doris Völker ${ }^{*}$
}

\begin{abstract}
Given the numerous manufactured nanomaterials already on the market and expected in future, the effort for the individual investigation of hazard and risk would be enormous. To overcome this challenge, grouping of nanomaterials has been identified as one critical issue by the European Commission as well as the Organisation for Economic Co-operation and Development (OECD). In that context, several research projects have been carried out to deliver scientific input and develop approaches. This publication discusses experiences from several cases of two scientific projects with the aims to develop and review analogy hypotheses for grouping of different nanoforms of same substances for endpoints related to aquatic ecotoxicity from a regulatory point of view. Furthermore, by presenting examples of grouping attempts, it outlines the need for considerations of further aspects beside key physical-chemical parameters for grouping nanomaterials regarding environmental endpoints. The results substantiate previous educated guesses with strategically collected experimental data on issues that needs to be considered for grouping nanomaterials in regulatory context. Still, grouping of nanomaterials for the aim of joint assessment of ecotoxicity of nanoforms in a regulatory context is in its infants compared to grouping approaches of nanoforms for human health assessment. This publication features important aspects on possibilities and challenges of grouping nanomaterials for environmental hazard assessment.
\end{abstract}

Keywords: Grouping, Nanomaterials, Nanoforms, Ecotoxicity, Algae, Daphnids

\section{Introduction}

Grouping and read-across/analogue approaches are already established for chemical substances to meet regulatory data requirements by deviation from performing standard test requirements for years [1,2]. The aim of these approaches is to predict the physical-chemical, toxicological, and fate-related properties of chemical substances based on structural similarities. If sufficient evidence is available, it should then be possible to transfer available data on the hazards of one chemical substance to another one. These approaches are intended to reduce the amount of testing necessary to determine

*Correspondence: kathrin.schwirn@uba.de; doris.voelker@uba.de German Environment Agency (Umweltbundesamt), Woerlitzer Platz 1, 06844 Dessau-Rosslau, Germany the behaviour and effects of all individual members of a group. Beside other benefits, these approaches will lead to a reduction of the number of experiments with animals.

In short, the utilisation of analogues is one opportunity to close data gaps within regulatory information requirements on hazard. This approach can be used to predict the outcome of individual or a number of endpoints provided that appropriate data are available. In doing so, information of a substance is used to make a qualitative or quantitative hazard statement for an endpoint under review (e.g., immobilisation of daphnia) for another substance, which shows similarities for that endpoint in important aspects like mode of action, toxico-kinetics, or metabolism. Prerequisite is that for the substance physicochemical properties, effects on human health and 
environment or fate and behaviour in the environment can be interpolated based on the data of the reference substance(s) of the same substance group. Similarities can be based on same functional groups, structurally similar products of the physical or biological degradation, or consistent pattern in the potency change of relevant properties across the substance group. According to the Annex XI of the Regulation for Registration, Evaluation, Authorisation and Restriction of Chemicals (REACH) grouping and read-across approaches can be used if following requirements are met: the results are adequate to classify and label a substance according to the Regulation for Classification, Labelling and Packaging of substances and mixtures (CLP) and/or to assess its risk [3, 4]; the results capture sufficiently the most important parameters which are specified by the endpoint relevant test method, and the exposure duration if relevant. The considered duration of the approach needs to be comparable to or longer than in the endpoint relevant test method, and the approach needs to be sufficiently and reliably documented.

For grouping and read across of nanomaterials, further aspects need to be considered. Here, the question concerns not only the transferability of data between different substances but also whether hazard data between different nanomaterials of the same chemical substance, the so-called nanoforms [5], can be transferred [6]. However, grouping and read across based on molecular structural similarity alone is not sufficient to develop and verify a grouping/read-across hypothesis that allows an adequate hazard assessment of nanomaterials while avoiding individual testing of a large number of the different nanomaterials. In that context, parameters like morphology, surface properties, and shape as well as reactivity, and fate descriptors like dissolution rate or dispersion stability in relevant media are discussed for consideration beside chemical composition [7]. Several research projects have been carried out to deliver scientific input and develop approaches.

Data used for this publication were already published in Hund-Rinke et al. [8] regarding the development of a grouping scheme for ecotoxicity. Physical-chemical properties relevant for ecotoxicity were identified. Based on these properties, it was possible to group various nanomaterials differing in their physical properties and chemical composition but showing comparable ecotoxicity. In addition, data originating from Kühnel et al. [9] on conceptional considerations for grouping nanomaterials regarding their fate and effects were used. The current publication discusses these data in sense of lessons learned for the development and confirmation of analogy hypotheses for grouping of different nanoforms of the same chemical substances for endpoints related to aquatic ecotoxicity from a regulatory perspective. Using data from aquatic ecotoxicity tests representing different trophic levels (algae and daphnia), important issues were identified that are crucial when applying grouping or read-across approaches for nanomaterials in a regulatory context. This analysis aims to raise awareness on the complexity of this issue and the specific needs for consideration for a valid grouping and analogue approach for nanoforms and ecotoxicity applied in a regulatory context. It highlights what are the possibilities and challenge of the illustrated cases. With this analysis, the scientific community obtains insight of the regulatory needs and regulators gain better understanding about the challenges of available data as basis for nanospecific grouping. Here, a situation is mirrored which regulators face in their daily work when assessing scientific data of different quality and quantity which need to be used for regulatory decision-making. The findings of this analysis support the relevance of the guidance for grouping of nanoforms given by the European Chemicals Agency (ECHA) and, at the same time, give a closer look on specific details which need to be considered for grouping and read across of nanomaterials for aquatic ecotoxicity. The paper will present considerations that should be taken into account for the establishing, verifying, and amending of grouping and analogy hypotheses of nanomaterials. The examples are based on the experiences form various silver (nano) forms and nanoforms of $\mathrm{TiO}_{2}, \mathrm{Fe}_{2} \mathrm{O}_{3}$, and $\mathrm{CeO}_{2}$ for the endpoints algae growth and daphnia immobilisation. As well, available characterisation and analytic information from the underlying projects of the respective publications were taken into account $[10,11]$. The nanoforms were chosen as they feature intrinsic and extrinsic properties which were hypothesised to be relevant for establishing groups and, thus, were deemed to be suitable for verifying grouping hypothesis.

\section{Information basis for approaching grouping hypotheses}

The data underlying this publication were collected in two different projects. These projects were two of the first projects dealing with strategic attempts for grouping nanoforms for environmental endpoints. Within these projects together, nearly 20 substances with different numbers of nanoforms were investigated for developing grouping approaches for various objectives, also those beyond regulatory use. For the present publication, data sets on nanoforms of four substances (silver, $\mathrm{CeO}_{2}, \mathrm{Fe}_{2} \mathrm{O}_{3}$, and $\mathrm{TiO}_{2}$ ) were chosen to highlight the challenges when building grouping and analogue hypotheses for nanomaterials and aquatic ecotoxicity. These examples were selected to develop, verify, and revise analogy hypotheses 
for grouping of chosen nanoforms for endpoints related to aquatic toxicity.

Three forms of silver were selected to (1) follow the hypothesis of a correlation between toxic ion release and increase in toxicity towards organisms as well as to (2) verify the hypothesis of shape influencing the toxicity towards organisms.

Additional examples were $\mathrm{CeO}_{2}, \mathrm{Fe}_{2} \mathrm{O}_{3}$, and $\mathrm{TiO}_{2}$ representing hardly soluble nanoforms varying in various physical chemical parameters (3). Three nanoforms of cerium dioxide $\left(\mathrm{CeO}_{2}\right)$ were chosen to investigate whether or not mainly primary particle size is triggering toxicity, while all other characteristics remain nearly identical. Three nanoforms of iron oxide $\left(\mathrm{Fe}_{2} \mathrm{O}_{3}\right)$ were used to investigate the influence of different shapes with similar primary particle sizes on ecotoxicity. Finally, the ecotoxicity of five nanoforms of titanium dioxide $\left(\mathrm{TiO}_{2}\right)$ was compared to figure out the similarity of effects based on doping and surface chemistry but also crystallinity.

The ecotoxicity of these (nano)forms was investigated using the standardised OECD test guidelines (TG) for determination of toxicity in algae and daphnids $[12,13]$. These tests were performed not to assess the hazard to the aquatic environment but to verify and/or revise the initial analogy hypotheses and approaches, which were developed based on comparison of intrinsic and extrinsic properties.

The physical chemical characteristics of the nanoforms, preparation of suspensions, the performance of characterisation, and ecotoxicological tests with algae and daphnids as well as the investigation of the attachment of nanoforms to the green algae $R$. subcapitata are described in $[8,9]$. In addition, for ionic silver and nanosilver data on the ecotoxicity on D. subspicatus were included as data for these forms were not generated with algae in the underlying projects [14]. Results on parameters considered as relevant for applying grouping for the investigated nanomaterials and resulting half maximal effective concentration $\left(\mathrm{EC}_{50}\right)$ values are shown in Tables 1 and 2. To relate ecotoxicity to the real concentration in the water phase during testing, the recovery of the total amount of silver for all investigated forms in the corresponding test media at the end of the testing is shown in Table 3. As no toxicity was observed for the respective nanoforms of $\mathrm{CeO}_{2}, \mathrm{Fe}_{2} \mathrm{O}_{3}$, and $\mathrm{TiO}_{2}$ to daphnids according to OECD TG 202, these data are not shown and discussed.

As initial step of this study for each of the substances, groups or representatives of groups of the regarded nanoforms were proposed based on extrinsic and intrinsic properties. Furthermore, hypotheses were built for the expected toxicity potentially based on these parameters. While building these hypotheses, it needs to be considered that more than one extrinsic or intrinsic property can trigger similarity or difference in toxicity of nanoforms. As first case groups for the different silver (nano)forms were proposed and qualitative differences of their toxic potentials towards daphnia and algae were estimated (Fig. 1). This case serves as an example for ion releasing substances as well as for substances of different shapes and a hypothesis for grouping was deduced. As a second case, nanoforms of poorly soluble $\mathrm{TiO}_{2}, \mathrm{Fe}_{2} \mathrm{O}_{3}$, and $\mathrm{CeO}_{2}$ were considered regarding defining of groups and their toxicity to algae (Fig. 2).

\section{Silver nanoforms: influence of shape and solubility on toxicity to algae and daphnia}

Up to now, the investigation of shape-related effects on environmental organisms is quite rare. Especially, compared to fibre-induced health effects, possible implications of the so-called high aspect ratio nanomaterials to environmental organisms are hardly reported. In this study, silver in different forms including wires, nanowires, and a spherical form were chosen to investigate the influence of shape on the potency to induce hazardous effects on aquatic organisms next to the influence of dissolution.

Data on dissolution behaviour after $24 \mathrm{~h}$ (data can be found in [10]) and $72 \mathrm{~h}$ in different media (OECD medium, ADaM medium, and water (data can be found in [10]) of the individual forms led to the assumption that the different silver forms represent 2-3 different groups based on the amount of released ions. Based on the total amount of dissolved ions, each form could represent an individual group. Based on the percentage portion of dissolved material, the (nano)wires could represent one group, while the spherical NM300K represent another group. It has to be taken into account that the data from these dissolution experiments might not be directly extrapolated to the dissolved fraction in the aquatic toxicity test as considerable lower concentrations of nano silver were applied for toxicity testing than for testing dissolution: It is assumed that the dissolved fraction (\% related to the nominal concentration) under the concentrations used in the aquatic toxicity test was higher than in the dissolution experiments [15]. However, total ion concentration in the test system can be lower or in the same range like in the performed dissolution experiment [16]. For the (nano)wires, microscopic images gave indication that a significant part was not dissolved in the test media but taken up by the daphnia [10]. Furthermore, low dissolution in the test media at test concentrations is also supported by the low-to-very low recovery of silver (nano)wires in the water phase (Table 3) indicating a low amount of available silver ions while suggesting strong sedimentation. 


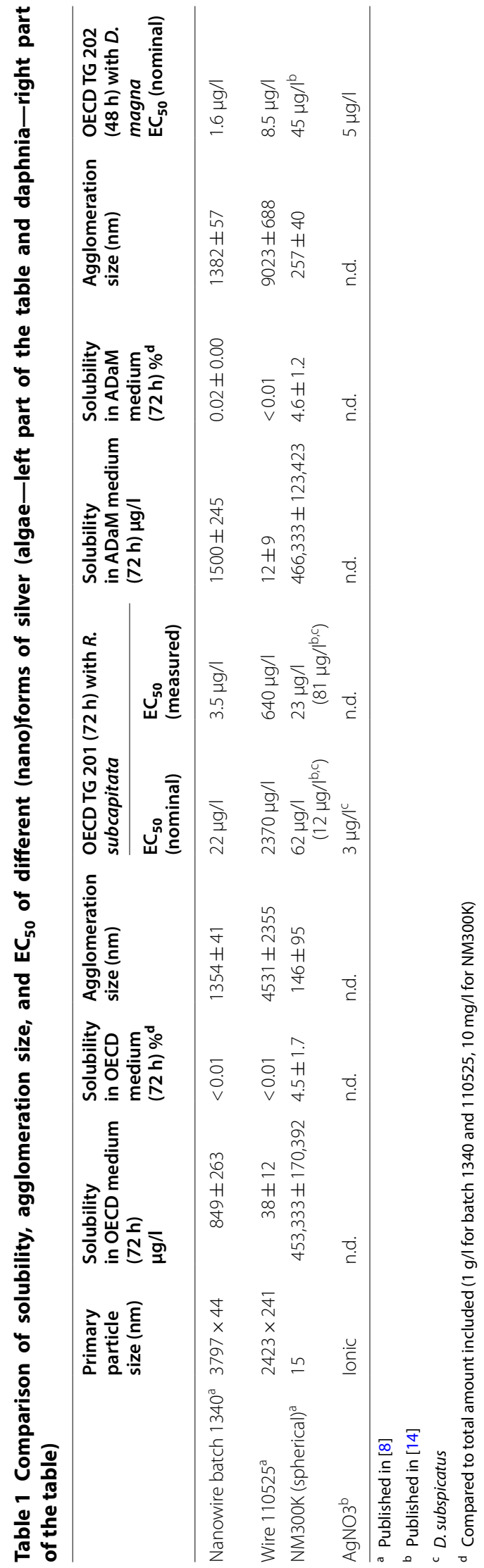




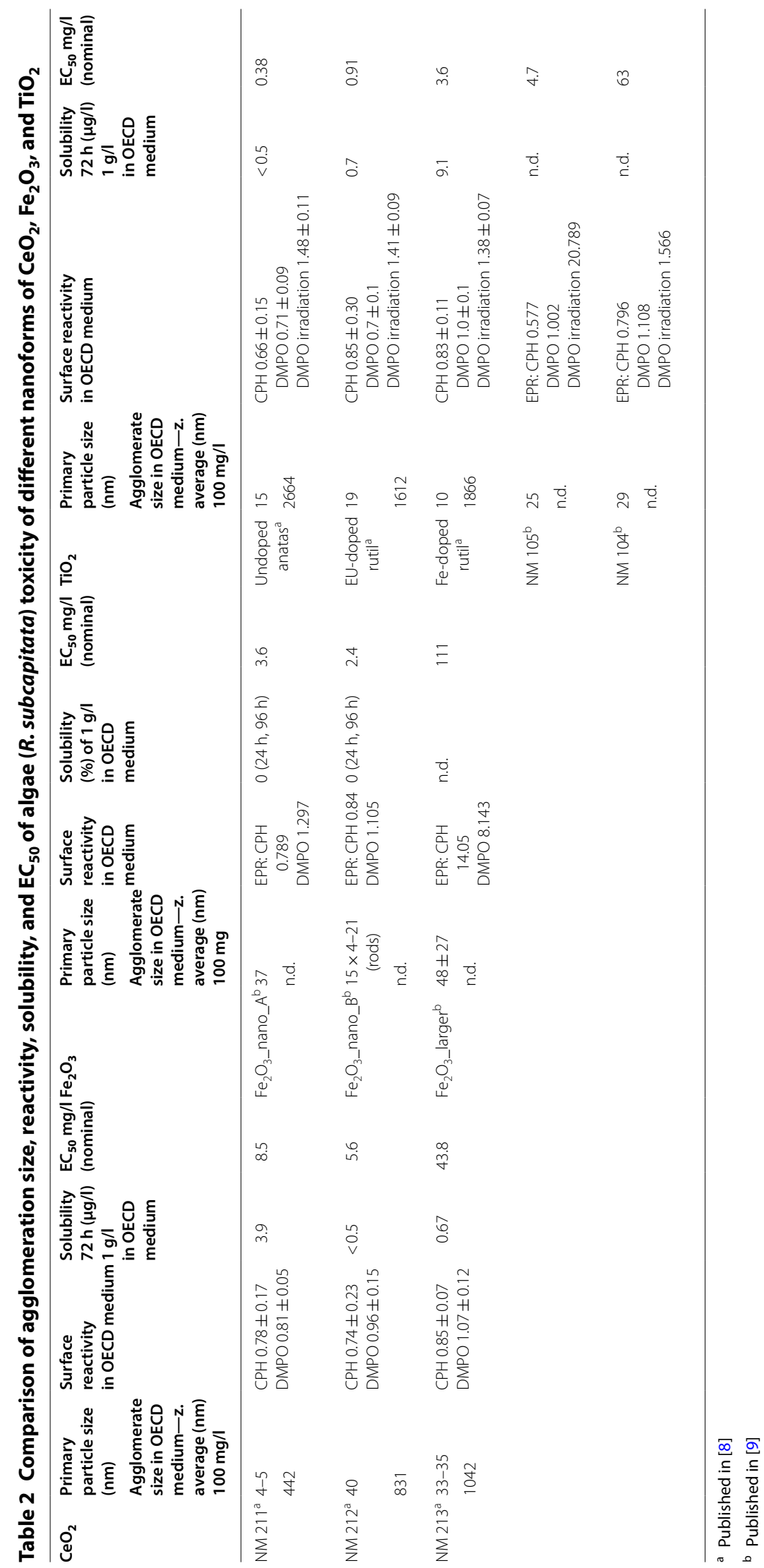


Table 3 Recovery of silver forms in medium of OECD TG 202 and 201 at the end of the testing

\begin{tabular}{|c|c|c|c|c|c|c|}
\hline & \multicolumn{3}{|c|}{ ADaM medium (acc. to OECD TG 202) } & \multicolumn{3}{|c|}{ OECD medium (acc. to OECD TG 201) } \\
\hline & $\begin{array}{l}\text { Nominal } \\
\text { concentration } \\
(\mu \mathrm{g} / \mathrm{l})\end{array}$ & $\begin{array}{l}\text { Analytical } \\
\text { concentration } \\
(\mu \mathrm{g} / \mathrm{l})\end{array}$ & Recovery (\%) & $\begin{array}{l}\text { Nominal } \\
\text { concentration } \\
(\mu \mathrm{g} / \mathrm{l})\end{array}$ & $\begin{array}{l}\text { Analytical } \\
\text { concentration } \\
(\mu \mathrm{g} / \mathrm{l})\end{array}$ & Recovery (\%) \\
\hline \multirow[t]{6}{*}{ Batch SRM 110525 (wire) } & 1 & $<0.1$ & $<0.1$ & 17 & 4.5 & 26 \\
\hline & 44 & 0.58 & 1.3 & 50 & 4.8 & 10 \\
\hline & 133 & 1.2 & 0.9 & 150 & 10 & 7 \\
\hline & 400 & 1.3 & 0.3 & 450 & 28 & 6 \\
\hline & & & & 1350 & 650 & 48 \\
\hline & & & & 4050 & 650 & 16 \\
\hline \multirow[t]{5}{*}{ Batch 1340 (nanowire) } & 1.6 & 0.88 & 55 & 1.9 & 0.1 & 5 \\
\hline & 4.9 & 1.1 & 22 & 5.6 & 0.5 & 9 \\
\hline & 14.8 & 1.8 & 12 & 17 & 2.5 & 15 \\
\hline & 44.4 & $<0.1$ & $<0.2$ & 50 & 12 & 24 \\
\hline & 400 & 33 & 8 & 150 & 40 & 27 \\
\hline \multirow[t]{5}{*}{ NM300K } & n.d. & n.d. & n.d. & 1.85 & 30 & $1622^{\mathrm{a}}$ \\
\hline & n.d. & n.d. & n.d. & 5.5 & 0.83 & 15 \\
\hline & n.d. & n.d. & n.d. & 16.5 & 1.2 & 7 \\
\hline & n.d. & n.d. & n.d. & 50 & 18 & 36 \\
\hline & n.d. & n.d. & n.d. & 150 & 68 & 45 \\
\hline
\end{tabular}

n.d. not defined

a Measuring error, the quality control of the chemical analysis shows no significant derivation

\section{Ag+}

$\begin{array}{llll}\text { Proposed groups dissolution } & \text { A } & \text { B } & \text { B or C } \\ \text { Proposed groups shape } & \text { A } & \text { B } & \text { B or C }\end{array}$

\section{Hypothesis based on dissolution}

\section{Expected toxicity due to ion release}

\section{Hypothesis based on shape}

\section{Expected toxicity due to availability}

Fig. 1 Initially proposed grouping of three different silver (nano)forms and expected toxicity to algae growth and daphnia immobilisation. While the representatives of the deduced groups based on the intrinsic and extrinsic properties seemed reasonable, hypothesis on their relative toxicological potential was not confirmed for every case [depicted silver (nano)forms from left to right: ionic form ( $\mathrm{AgNO}_{3}$ ), NM300K, Batch 1340 (nanowire), 110525 (wire)] 


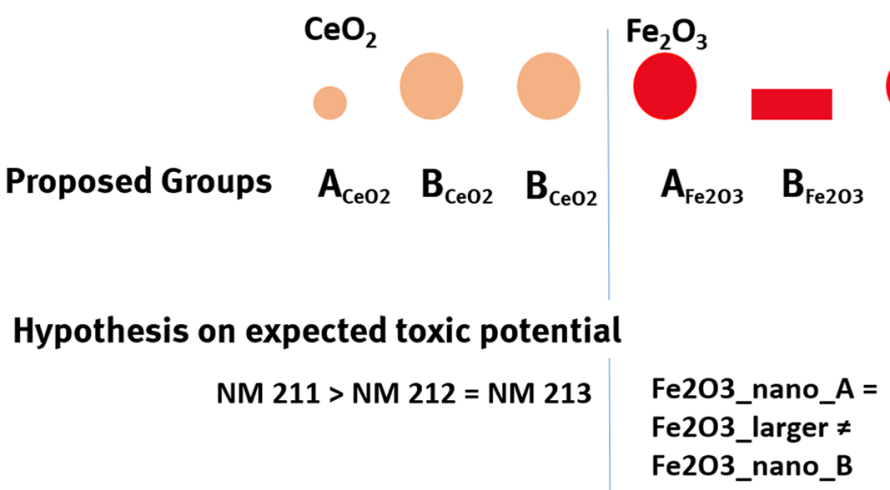

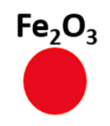

$A_{\text {fe203 }}$
$\mathrm{TiO}_{2}$

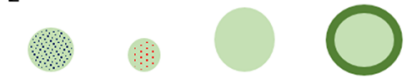

$\begin{array}{lllll}\mathbf{A}_{\mathrm{TiO} 2} & \mathbf{A}_{\mathrm{TiO} 2} & \mathbf{A}_{\mathrm{TiO} 2} & \mathbf{B}_{\mathrm{TiO} 2} & \mathbf{A}_{\mathrm{TiO} 2}\end{array}$

(alternatively: each nanoform presents an own group)

Toxic potential in the same range except NM 105

Fig. 2 Initially proposed grouping of different (nano)forms of $\mathrm{CeO}_{2}, \mathrm{Fe}_{2} \mathrm{O}_{3}$, and $\mathrm{TiO}_{2}$, and hypotheses on expected toxic potential to algae growth. The assumed qualitative differences in the toxicological potential were not confirmed and further aspects than initially considered need to be included for final conclusions [depicted nanomaterials from left to right- $\mathrm{CeO}_{2}: \mathrm{NM} 211, \mathrm{NM} 212, \mathrm{NM} 213 ; \mathrm{Fe}_{2} \mathrm{O}_{3}: \mathrm{F}_{2} \mathrm{O}_{3} \_$nano_A, $\mathrm{F}_{2} \mathrm{O}_{3} \_$nano_B $\mathrm{F}_{2} \mathrm{O}_{3}$ nano_larger; $\mathrm{TiO}_{2}$ : undoped anatase, Eu-doped rutil, Fe-doped rutil, NM 105, NM 104]

Based on the shape and the agglomeration size in the respective test media (see Table 1), it was expected that the availability of the (nano)wires would be considerably lower than of the spherical nanoform. Thus, it was conceivable to rank the wire forms into one or two separate group(s), either both forms together or divided into a nano and a non-nano group next to a postulated group represented here with the spherical NM300K likewise based on solubility.

Based on the determined shape and solubility of the individual silver forms, it was hypothesised that the spherical form should be considerable more toxic to algae and daphnids than the (nano-)wires (Fig. 1). This hypothesis was derived by the assumptions that the toxicity increases with increasing amount of available silver ions as well as on a low availability of the (nano)wires to the test organisms. However, data on daphnids (based on OECD TG 202) and algae (based on OECD TG 201) did not confirm that hypothesis on toxicity. No correlation between ion release and the toxicity in algae and daphnia was observed. For example, despite NM300K showed highest ion release, silver (nano)wires were more toxic to daphnids.

For daphnids, the (nano-)wires showed $\mathrm{EC}_{50}$ values based on nominal concentrations (see Table 1) which were one magnitude lower than for NM300K, however, in the same order of magnitude like the ionic form with a slightly lower $\mathrm{EC}_{50}$ for the nanowire (batch 1340). In addition, when considering the analytic concentration at the end of the test (see Table 3), the analytic $\mathrm{EC}_{50}$ values for the (nano)wires and NM300K should be even lower. Based on that data, the toxicity of the silver (nano)wires cannot be fully explained by toxicity of the ions. In consequence, the proposed differentiation between spherical and fibre like forms seems not to be contradicted by the results of the acute toxicity tests of daphnia, but the hypothesis to predict the toxic potential of the different groups based on ion release and shape could not be verified. Furthermore, the ionic form does not seem to represent a worst case for the nanowires. Microscopic investigation of the exposed daphnids showed that a considerable amount of (nano)wires were taken up into the gut. It is conceivable that additional mechanical effects (e.g., blocking or impairment of the digestive tract) and/ or local increase in ion release due to changing conditions in the gut system compared to test media could lead to the observed increased toxicity to the daphnids.

The results for algae showed (Table 1) highest toxicity of the ionic form based on nominal concentration followed by the nanowire (batch 1340), the spherical nanosilver NM300K, and the wire (batch SRM 110525). However, when considering measured concentrations, the $\mathrm{EC}_{50}$ of the nanowire (batch 1340) is in the same order of the $\mathrm{EC}_{50}$ of ionic form, while effect values for spherical NM300K and the silver wire (batch SRM 110525) were one and two order of magnitude higher, respectively. Thus, for algae toxicity, it seems verified that the spherical nanosilver NM300K represents a different group than the nanowire, but, again, the hypothesis to predict the deviating toxicity potential of the different groups could not be fully verified. The ionic form can represent a worst case for the investigated silver (nano)forms regarding algae toxicity. Nevertheless, the available data of the solubility test and recovery do not indicate whether the toxic effects are fully explainable by the release of silver ions. Microscopic images gave evidence that, for the (nano) wires, a significant part was still undissolved and images with the nanowire (batch 1340) gave indication that it 
interacts with the algae [10]. Thus, mechanical effects of the nanowire due to skewing and/or local increased ion release at the algae cell wall could be a possible explanation for an increased toxicity of the nanowires compared to the wires or NM300K, respectively. For the wire (batch SRM 110525), it remains, thus, unclear if it represents another group than the nanowire (batch 1340) based on the difference in toxicity or if it is part of the same group considering that, within the group, a gradient of toxicity can appear. To properly assign these forms into one or different groups, more examples of such nano(forms) need to be included in the examination.

Comparing the toxicity of NM300K on growth of the algae species $R$. subcapitata and for D. subspicatus, differences in the toxic response can be observed. This might be caused by inter-laboratory variation or differences in sensitivities of both species to nanomaterials. Higher sensitivity of $R$. subcapitata compared to D. subspicatus to nanomaterials was observed for different nanoforms of $\mathrm{Fe}_{2} \mathrm{O}_{3}$ and $\mathrm{SiO}_{2}$ or for metal compounds $[9,17]$.

\section{Nanoforms of $\mathrm{TiO}_{2}, \mathrm{CeO}_{2}$, and $\mathrm{Fe}_{2} \mathrm{O}_{3}$ : Influence of agglomeration, reactivity, and solubility on toxicity to algae}

In this study, also poorly soluble nanomaterials (different nanoforms of $\mathrm{TiO}_{2}, \mathrm{CeO}_{2}$, and $\mathrm{Fe}_{2} \mathrm{O}_{3}$ ) were investigated to better understand the relevance of the parameters size, agglomeration size, reactivity, and solubility on grouping. Differences in toxicity might arise due to the variance in the primary particle size of $\mathrm{CeO}_{2}$ nanoforms NM 211 compared to NM 212 and NM 313 and the rod like $\mathrm{Fe}_{2} \mathrm{O}_{3-}$ nano_B compared to the spherical $\mathrm{Fe}_{2} \mathrm{O}_{3-}$ nano_A and $\mathrm{Fe}_{2} \mathrm{O}_{3-}$ larger. For $\mathrm{TiO}_{2}$, the situation is more complex. On one hand, some of the nanoforms have comparable sizes: e.g., the undoped anatase and the Fe-doped $\mathrm{TiO}_{2}$ or the NM104 and NM105, respectively. However, on the other hand, all forms considerably differ due to their crystallinity, surface chemistry, or doping. However, a closer look on agglomeration size, reactivity, and solubility do not show clear differences or trends except the reactivity on NM 105 under irradiation. Proposed groups of the different nanoforms of $\mathrm{TiO}_{2}, \mathrm{CeO}_{2}$, and $\mathrm{Fe}_{2} \mathrm{O}_{3}$ and hypotheses on the expected toxic potential to algae can be found in Fig. 2.

Toxicity testing of algae revealed $\mathrm{EC}_{50}$ values differing between one and three orders of magnitudes between the different nanoforms of all three substances and did not confirm the hypotheses for the expected toxicity (see Table 2). Substance-wise, a relationship between toxicity data and data on primary particle size as well as on solubility, reactivity, and data on agglomeration size in the test media for these nanoforms could not be found. To screen for alternative explanation for toxicity, i.e., an attachment of the various nanoforms to algae cells, a growing culture of algae was exposed to a $100 \mathrm{mg} / \mathrm{L}$ suspension for 1 or $3 \mathrm{~h}$ [9].

In the Additional Information of [9], examples of different nanoforms of $\mathrm{CeO}_{2}, \mathrm{Fe}_{2} \mathrm{O}_{3}$, and $\mathrm{TiO}_{2}$ interacting with algae cells are presented. For $\mathrm{CeO}_{2}$ and $\mathrm{Fe}_{2} \mathrm{O}_{3}$, the figures demonstrate lower particle attachment to the algae of those nanoforms which feature higher $\mathrm{EC}_{50}$ values $\left(\mathrm{CeO}_{2}\right.$ NM 213 and $\mathrm{Fe}_{2} \mathrm{O}_{3}$ larger, respectively). For nanoforms with lower $\mathrm{EC}_{50}$, an extensive interaction between particles and algae cells is obvious. Thus, for the investigated $\mathrm{CeO}_{2}$ and $\mathrm{Fe}_{2} \mathrm{O}_{3}$, interaction with algae cells seems to drive toxicity differences of nanoforms. Attachment of nanomaterials to algae might be explained by exudates which are exceeded by algae under stress. Furthermore, investigations show that exudate compositions vary with algae species [18]. This might also explain differences in sensitivity of distinct species of algae as depicted above.

In contrast to this observation, the reasons for differences in algae toxicity of $\mathrm{TiO}_{2}$ forms seem to be more complex. A direct relation of the toxic potential with primary particle size, agglomeration size in test media, crystallinity, or the presence of a doping could not be observed. Even more, NM 105 which showed highest reactivity under DMPO (5,5-dimethyl-1-pyrroline) and irradiation was not the most toxic $\mathrm{TiO}_{2}$ nanoform under investigation.

Within the attachment tests, except from the undoped anatase nanoform, all investigated $\mathrm{TiO}_{2}$ nanoforms visibly form big agglomerates which settle to the bottom of the vessel, with NM 104 showing the biggest agglomerates. These differences in behaviour are reflected in the $\mathrm{EC}_{50}$ values showing the lowest $\mathrm{EC}_{50}$ for the undoped anatase nanoform and a two magnitudes of order higher $\mathrm{EC}_{50}$ for NM104 (165-fold compared to the anatase nanoform) featuring the highest $\mathrm{EC}_{50}$ of the investigated $\mathrm{TiO}_{2}$ nanoforms. Still, the agglomeration alone did not explain the differences or sameness in the $\mathrm{EC}_{50}$ values for the other forms. The Eu-doped rutil nanoform with an $\mathrm{EC}_{50}$ value in the same range of the undoped anatase form shows likewise a strong interaction between algae and the particles; the algae are completely covered. For the Fe-doped rutil nanoform which features $\mathrm{EC}_{50}$ value of one magnitude higher than the Eu-doped rutil nanoform, the coverage is less pronounced; still, some of the observed algae cells are fully covered by the particles. NM 104 shows strong agglomeration in the test system and attachment to the algae is less pronounced compared to the already described forms. This nanoform shows the highest $\mathrm{EC}_{50}$ value of the investigated $\mathrm{TiO}_{2}$ nanoforms. Based on visual examination of the pictures presenting the attachment of NM 105 to algae [9], the degree of attachment is questionable; still, the $\mathrm{EC}_{50}$ value is one 
order of magnitude lower than for NM 104. However, as mentioned above, NM 105 showed a higher reactivity in DMPO measurements, which may indicate that despite the lower attachment compared to the anatase or doped $\mathrm{TiO}_{2}$ nanoforms, NM 105 could cause higher toxic effects due to its surface reactivity.

In consequence, the assumed qualitative differences in the toxicological potential for the investigated nanoforms of $\mathrm{CeO}_{2}, \mathrm{Fe}_{2} \mathrm{O}_{3}$, and $\mathrm{TiO}_{2}$ were not confirmed. Here, further aspects like attachment of the nanomaterials to algae need to be considered to improve grouping hypotheses. For $\mathrm{CeO}_{2}$ and $\mathrm{Fe}_{2} \mathrm{O}_{3}$, this assumption seems more reasonable than for $\mathrm{TiO}_{2}$. However, the diversity of $\mathrm{TiO}_{2}$ nanoforms is higher which leads to more complexity.

\section{Conclusions}

Grouping of nanoforms of a substance with the aim to transfer available data on a hazard endpoint from one nanoform to another one is one major tool to address the challenges in the assessment of countless nanomaterials. While the understanding of grouping of nanomaterials for comparable toxic effects on human health considerably progressed, analogous principles for environmental health are still at the beginning. This evaluation tried to build and verify grouping hypothesis for selected nanoforms of the same substances based on available data from two research projects to investigate the relevance of physical chemical properties as indicated, e.g., by ECHA guidance. Even though it was not possible to deduce a final conclusion for valid grouping for the considered cases yet, the results support the guidance given by ECHA that, beside key parameters like solubility, shape, and agglomeration, other aspects like interaction with the investigated organism need to be taken into account. So far, assumptions on aspects relevant for grouping and analogy approaches for nanomaterials on environmental endpoints for regulatory use mainly based on educated guesses deduced from a synopsis of available data on environmental fate and effects. These are now substantiated with strategically collected experimental data. Still, the results indicate that the prediction of the ecotoxicological potential of nanoforms remains very challenging due to the interplay of various intrinsic and extrinsic properties of nanoforms and thereby limited.

Several aspects were identified that need to be considered for deriving grouping hypotheses of nanoforms based on aquatic toxicity:

i. Dissolution, agglomeration, and sedimentation are important descriptors to understand the fate and uptake routes of nanomaterials in the test system. However, it is inappropriate to exclude the occur- rence of hazard only based on this information as agglomeration and sedimentation or insolubility do not automatically prevent availability to or uptake by organisms.

ii. For certain nanomaterials, the attachment to algae seems important for the prediction of adverse effects. Based on the presented data, the attachment to algae is not directly predictable from the measured agglomeration size or observed settling behaviour. Changed conditions at the surface of the organisms due to, e.g., exudates may alter nanomaterial behaviour and, consequently, lead to an attachment to the algae depending on nanomaterial properties.

iii. The chemical milieu inside the organism (e.g., gut) or high internal concentration of nanomaterials may lead to an increased local ion release that is not apparent from the solubility in the test media.

iv. Mechanical impacts leading to a blocking of organisms organs or impairment of physiological processes may contribute to the observed effect and, therefore, need to be considered for grouping hypothesis.

v. To be able to identify a potential gradient of toxicity within a group and to define group boundaries, more nanoforms than examined in this study with slightly changing parameters need to be investigated.

For the scope of this evaluation (building and revising grouping hypothesis ecotoxicity of nanoforms based on intrinsic and extrinsic properties), it became obvious that essential analytical data are missing, e.g., time-dependent characterisation of dissolution kinetics. The relevance of the individual properties for grouping can neither be ranked nor judged based on consideration of only one single parameter or jointly deduced for all ecotoxicological endpoints. Therefore, the defined groups and toxicity hypotheses still need to be verified by further investigation and care should be taken on which information a hypothesis is based on. Furthermore, for other endpoints, substances, or nanoforms other outcomes than achieved in the presented projects are conceivable as grouping and analogue approaches in regulatory context are case-bycase considerations.

In the current situation, it needs to be carefully evaluated for the individual case under consideration how much effort should be made performing testing for a robust and reliable grouping hypothesis compared to the effort to directly determine the effect data for ecotoxicity of individual nanoforms. 


\begin{abstract}
Abbreviations
OECD: Organisation for Economic Co-operation and Development; REACH: Regulation for Registration, Evaluation, Authorisation and Restriction of Chemicals; CLP: Regulation for Classification, Labelling and Packaging of substances and mixtures; ECHA: European Chemicals Agency; TG: test guideline; $\mathrm{EC}_{50}$ : half maximal effective concentration; DMPO: 5,5-dimethyl1-pyrroline; EPR: Electron paramagnetic resonance spectroscopy; $\mathrm{CPH}$ : 1-hydroxy-3-carboxy-pyrrolidine.
\end{abstract}

\section{Acknowledgements}

The authors thank Kerstin Hund-Rinke, Dana Kühnel, and Carmen Nickel for project co-operation, fruitful discussion, and critical review of the manuscript

\section{Declarations}

The views expressed in this manuscript are those of the authors and may not necessary be regarded as official position of the German Environment Agency.

\section{Authors' contributions}

All authors analysed and interpreted the mentioned data and wrote the manuscript. All authors read and approved the final manuscript.

\section{Funding}

This work was supported by the German Federal Ministry of Education and Research within the research project NanoGRAVUR (Grant No.: 03XP0002) and by the German Federal Ministry for the Environment, Nature Conservation and Nuclear Safety within the ReFoPlan Project FKZ 3714674170 which was supervised by the German Environmental Agency. The central aim of nanoGRAVUR was to develop criteria catalogues for a grouping of nanomaterials regarding hazard and risk potentials related to different subjects of protection (consumer, worker, and environment). The German Environmental Agency was project partner in nanoGRAVUR with the task inter alia to evaluate the project outcomes for regulatory applicability. Aim of the ReFoPlan Project FKZ 3714674170 was to correlate physical-chemical data with ecotoxicological effects for selected nanomaterials and try to define reference values which can serve as a basis for grouping.

\section{Availability of data and materials}

All data generated or analysed during this study are included in the published articles $[8-10,14]$ and their additional information files.

\section{Ethics approval and consent to participate}

Not applicable.

\section{Consent for publication}

Not applicable.

\section{Competing interests}

The authors declare that they have no competing interests.

\section{Received: 19 March 2019 Accepted: 28 June 2019}

Published online: 30 July 2019

\section{References}

1. European Chemicals Agency (2008) Guidance on information requirements and chemical safety assessment. Chapter R.6: QSARs and grouping of chemicals. http://echa.europa.eu/documents/10162/13632/informatio n_requirements r6 en.pdf. Accessed 30 Jan 2019

2. Organisation for Economic Co-operation and Development (2007) OECD Guidance on Grouping of Chemicals, Series on Testing \& Assessment No. 80. ENV/JM/MONO(2007)28. http://www.oecd.org/officialdocuments/ publicdisplaydocumentpdf/?cote=env/jm/mono(2007)28\&doclanguag e=en. Accessed 30 Jan 2019

3. Regulation (EC) No 1907/2006 of the European Parliament and of the Council of 18 December 2006 concerning the Registration, Evaluation, Authorisation and Restriction of Chemicals (REACH), establishing a European Chemicals Agency, amending Directive 1999/45/EC and repealing
Council Regulation (EEC) No 793/93 and Commission Regulation (EC) No 1488/94 as well as Council Directive 76/769/EEC and Commission Directives 91/155/EEC, 93/67/EEC, 93/105/EC and 2000/21/EC. https://eur-lex. europa.eu/eli/reg/2006/1907/?locale=en. Accessed 30 Jan 2019

4. Regulation (EC) No 1272/2008 of the European Parliament and of the Council of 16 December 2008 on classification, labelling and packaging of substances and mixtures, amending and repealing Directives 67/548/ EEC and 1999/45/EC, and amending Regulation (EC). https://eur-lex. europa.eu/eli/reg/2008/1272/?locale=en. Accessed 30 Jan 2019

5. Commission Regulation (EU) 2018/1881 of 3 December 2018 amending Regulation (EC) No 1907/2006 of the European Parliament and of the Council on the Registration, Evaluation, Authorisation and Restriction of Chemicals (REACH) as regards Annexes I, III, VI, VII, VIII, IX, X, XI, and XII to address nanoforms of substances. http://data.europa.eu/eli/ reg/2018/1881/oj. Accessed 30 Jan 2019

6. Schwirn K, Tietjen L, Beer I (2014) Why are nanomaterials different and how can they be appropriately regulated under REACH? Environ Sci Europe 26:4. https://doi.org/10.1186/2190-4715-26-4

7. European Chemicals Agency (2017) Guidance on information requirements and chemical safety assessment, Appendix R.6-1 for nanomaterials applicable to the guidance on QSARs and grouping of chemicals. https ://echa.europa.eu/documents/10162/23036412/appendix_r6_nanom aterials_en.pdf/71ad76f0-ab4c-fb04-acba-074cf045eaaa. Accessed 30 Jan 2019

8. Hund-Rinke K, Schlich K, Kühnel D, Hellack B, Kaminski H, Nickel C (2018) Grouping concept for metal and metal oxide nanomaterials with regard to their ecotoxicological effects on algae, daphnids and fish embryos. Nanolmpact 9:52-60. https://doi.org/10.1016/j.impact.2017.10.003

9. Kühnel D, Nickel C, Hellack B, van der Zalm E, Kussatz C, Herrchen M, Meisterjahn B, Hund-Rinke K (2019) Closing gaps for environmental risk screening of engineered nanomaterials. Nanolmpact. https://doi. org/10.1016/j.impact.2019.100173

10. Hund-Rinke K, Nickel C, Kühnel D (2017) Considerations about the relationship of nanomaterial's physical-chemical properties and aquatic toxicity for the purpose of grouping. In: UBA TEXTE 102/2017. https:// www.umweltbundesamt.de/publikationen/considerations-about-therelationship-of

11. Joint project nanoGRAVUR (Grant No.: 03XP0002) sponsored by the German Federal Ministry of Education and Research (BMBF)

12. Organisation for Economic Co-Operation and Development (2011) OECD test guideline 201: freshwater alga and cyanobacteria, growth inhibition test. https://doi.org/10.1787/9789264069923-en

13. Organisation for Economic Co-Operation and Development (2004) OECD test quideline 202: Daphnia sp. acute immobilisation test. https://doi. org/10.1787/9789264069947-en

14. Voelker D, Schlich K, Hohndorf L, Koch W, Kuehnen U, Polleichtner C, Kussatz C, Hund-Rinke K (2015) Approach on environmental risk assessment of nanosilver released from textiles. Environ Res 140:661-672. https://doi. org/10.1016/j.envres.2015.05.011

15. Baek YW, An YJ (2011) Microbial toxicity of metal oxide nanoparticles $\left(\mathrm{CuO}, \mathrm{NiO}, \mathrm{ZnO}\right.$, and $\left.\mathrm{Sb}_{2} \mathrm{O}_{3}\right)$ to Escherichia coli, Bacillus subtilis, and Streptococcus aureus. Sci Total Environ 409:1603-1608. https://doi.org/10.1016/j. scitotenv.2011.01.014

16. Liu J, Hurt R (2010) lon release kinetics and particle persistence in aqueous nano-silver colloids. Environ Sci Technol 44:2169-2175. https://doi. org/10.1021/es9035557

17. Rojíčková R, Maršálek B (1999) Selection and sensitivity comparisons of algal species for toxicity testing. Chemosphere 38:3329-3338. https://doi. org/10.1016/S0045-6535(98)00566-9

18. Marsálek B, Rojíčkovâ R (1996) Stress factors enhancing production of algal exudates: a potential self-protective mechanism? Zeitschrift fur Naturforschung-Section C J Biosci 51:646-650

\section{Publisher's Note}

Springer Nature remains neutral with regard to jurisdictional claims in published maps and institutional affiliations. 